\title{
A Real-World Assessment of Unvaccinated And Vaccinated Patients With Coronavirus Disease 2019 (COVID-19) Treated With Monoclonal Antibodies During The Delta Wave
}

Yi Guo ( $\nabla$ yiguo@montefiore.org )

Montefiore Medical Center

Kelsie Cowman

Montefiore Medical Center

Mei Chang

Montefiore Medical Center

Hongkai Bao

Montefiore Medical Center

Austin Golia

Montefiore Medical Center

Terrence Mcsweeney

Montefiore Medical Center

Linda Bard

Montefiore Medical Center

Roxanne Simpson

Montefiore Medical Center

Erin Andrews

Montefiore Health System

Liise-anne Pirofski

Montefiore Medical Center

Priya Nori

Montefiore Medical Center

\section{Research Article}

Keywords: Coronavirus disease 19, COVID-19, Delta variant, SARS-CoV-2 variant B.1.617.2, monoclonal antibody, casirivimab/imdevimab, vaccination, vaccinated, unvaccinated.

Posted Date: February 8th, 2022 
DOI: https://doi.org/10.21203/rs.3.rs-1289174/v1

License: (c) (1) This work is licensed under a Creative Commons Attribution 4.0 International License. Read Full License 


\section{Abstract}

Background: Monoclonal antibodies (mAb) prevent COVID-19 progression during early disease presentation. We aimed to compare the mAb treatment outcomes among vaccinated and unvaccinated patients during Delta wave and assess the feasibility of implementing stricter eligibility criteria given mAb scarcity in New York City.

Methods: We conducted a retrospective observational study of casirivimab/imdevimab recipients with mild-to-moderate COVID-19 infection in an emergency department or outpatient infusion center (July 1 August 20, 2021). The outcome was all-cause hospital admission within 30 days post-treatment between vaccinated vs. unvaccinated patients during Delta surge in the Bronx, NY.

Results: A total of 250 patients received casirivimab/imdevimab (162 unvaccinated vs. 88 vaccinated). The median age was 39 years for unvaccinated patients, and 52 years for vaccinated patients $(p<0.0001)$. The median number of EUA criteria met was 1 for unvaccinated and 2 for vaccinated patients $(p<0.0001)$. Overall, $6 \%(15 / 250)$ of patients were admitted within 30 days post-treatment. Eleven unvaccinated patients (7\%) were admitted (all-cause) within 30-days compared to $4(5 \%)$ of vaccinated patients $(p=0.48)$.

Conclusions: All-cause 30-day admission was not statistically different between vaccinated and unvaccinated patients. With limited federal allocation of therapies, programs must prioritize patients at highest risk of hospitalization and death regardless of vaccination status.

\section{Background}

Multiple studies demonstrate the efficacy of monoclonal antibodies (mAb) in preventing progression of COVID-19 when administered to high-risk patients early in disease presentation. ${ }^{1-6} \mathrm{~A}$ study by Cooper and colleagues evaluated nearly 3000 patients with coronavirus disease 2019 (COVID-19) who received mAb therapies in Houston, TX. ${ }^{7}$ Compared to a propensity-matched control cohort, mAb-treated patients had significantly lower rates of hospitalization at 14 - and 28-days and decreased mortality at 28-days postinfusion. ${ }^{7}$ However, the impact of circulating SARS-CoV-2 variants was not addressed. A study conducted at our medical center in the Bronx, NY revealed a concerning increase in bamlanivimab treatment failures between January and April 2021, likely due to an increase in the locally circulating variant B.1.526, which was resistant to bamlanivimab monotherapy. ${ }^{8}$ To date, there is a paucity of data addressing regional heterogeneity, outcomes during the Delta surge, or vaccination status of mAb-treated COVID-19 patients. The SARS-CoV-2 variant B.1.617.2 was identified in $70-98 \%$ of sequenced isolates across multiple New York City zip codes until July $2021 .{ }^{9}$ Despite the high efficacy of mRNA vaccines against severe disease and hospitalization, an increasing number of breakthrough SARS-CoV-2 infections due to the Delta variant were reported ${ }^{10,11}$, potentially due to waning antibody levels. ${ }^{12}$ The Bronx has had lower vaccine uptake among New York City boroughs. Its residents remain particularly vulnerable to SARS-CoV-2 infection ${ }^{13}$ and access to COVID-19 mAb therapies is of great importance here. 
In December 2020, our antimicrobial stewardship team (AST) established a COVID-19 infusion program to treat high-risk patients in the Bronx as per Food and Drug Administration (FDA) emergency use authorization (EUA) criteria. ${ }^{8}$ In May 2021, the FDA expanded treatment criteria by lowering the body mass index (BMI) threshold from $35 \mathrm{~kg} / \mathrm{m}^{2}$ to $25 \mathrm{~kg} / \mathrm{m}^{2}$ and including additional comorbidities. ${ }^{14,15}$ Importantly, patients with history of COVID-19 vaccination are not excluded. In September 2021, the US Health and Human Services Administration modified the mAb allocation process whereby states with fewer COVID-19 infections would receive fewer doses than states with high SARS-CoV-2 transmission. ${ }^{16}$ The new federal strategy impacted workflows of programs serving communities with high SARS-CoV-2 transmission and lower vaccine uptake.

The primary objective of this study was to evaluate mAb treatment outcomes among vaccinated and unvaccinated patients during the Delta surge in the Bronx, NY. The secondary objective was to assess the feasibility of implementing stricter mAb eligibility criteria given the impending resource scarcity in New York City.

\section{Methods}

We conducted a retrospective observational study of patients with mild-to-moderate COVID-19 meeting expanded EUA criteria received casirivimab/imdevimab in an emergency department (ED) or outpatient infusion center at Montefiore Medical Center (MMC) between July 1 - August 20, 2021. ${ }^{14-15}$ Our primary outcome was all-cause hospital admission 30 days post-treatment. COVID-19-related admissions were defined as known symptoms or sequelae (e.g., worsening respiratory status or thromboembolic complications), as determined by the study team. ${ }^{8}$ Patients were considered fully vaccinated two weeks following receipt of 2 doses of BNT162b2 or mRNA-1273, or 1 dose of Ad26.COV2.S.

Immunocompromise was defined as having HIV, liver cirrhosis, sickle cell anemia, asplenia, malignancy, solid organ transplant, bone marrow transplant, active chemotherapy treatment within the past 30 days, chronic use of steroids, tacrolimus, mycophenolate, or biologics. ${ }^{8}$ Institutional prioritization schema utilized September 2021 onward is shown in table 1.

\section{Table 1: Institutional Prioritization Schema for Monoclonal Antibody Therapy during the Delta Wave}


Unvaccinated/partially vaccinated AND symptomatic $\leq 7$ days*
Immediate treatment (Emergency

Department or same day in infusion center)
Next day scheduling at infusion center

Immediate treatment (Emergency Department or same day in infusion center)
Next day referral at infusion center

\section{Unvaccinated/partially vaccinated AND asymptomatic}

Wait and watch, reassess for treatment in $48 \mathrm{~h}$ if symptom progression

\section{Fully vaccinated AND asymptomatic}

\section{Fully vaccinated AND mildly symptomatic $\leq 7$ days*}

\section{Hold off on treatment}

Wait and watch, reassess for treatment in $48 \mathrm{~h}$ if symptom progression

If sufficient doses available for PEP, treat in ED or other nonCOVID location as soon as possible; requires same-day negative SARS-CoV-2 PCR)

\section{Post-exposure prophylaxis (PEP) (recent} significant exposure in high-risk unvaccinated patient OR severely immunocompromised patient without recent booster or no documented response by serologic testing)

*Patients with immune compromise, suspected poor response to vaccines, and potential prolonged viral shedding can be treated up to 10 days from symptom onset per Emergency Use Authorization criteria; use SARS-CoV-2 PCR cycle threshold (Ct) value (if available) to help guide clinical judgement; ONLY use Ct value in clinical context

Patients admitted due to COVID-19 progression on the same day as mAb treatment were excluded. ${ }^{8}$ Bivariate analyses were conducted using $X^{2}$, Fisher's exact test, or $t$ test, as appropriate. Multivariable analysis was conducted using logistic regression. All statistical tests were 2-tailed and $P<0.05$ were considered significant. Analyses were conducted using SAS, version 9.4 software (SAS Institute, Cary, NC). The Albert Einstein College of Medicine institutional review board approved the study with waiver of informed consent (IRB\# 2020-12525)

\section{Results}

Between July 1 - August 20, 2021, 250 COVID-19 patients received casirivimab/imdevimab; 162 (65\%) were unvaccinated and $88(35 \%)$ were vaccinated at time of treatment. The median age was 39 years for unvaccinated patients, and 52 years for vaccinated patients $(p<0.0001)$. Overall, $101(40 \%)$ of patients were Hispanic, 89 (36\%) non-Hispanic Black, and 31 (12\%) non-Hispanic white. Median BMI was not significantly different between the unvaccinated (30) and vaccinated (30) groups ( $p=0.87)$. Vaccinated patients had shorter duration of COVID-19 symptoms prior to treatment (median 3 vs. 4 days, $p=0.02$ ). The most common risk factor was BMI >25 kg/m², observed in 115 (71\%) unvaccinated and 67 (76\%) 
vaccinated patients $(p=0.66)$. The median number of EUA criteria met was 1 for unvaccinated and 2 for vaccinated patients $(p<0.0001)$, table 2 . Cycle threshold $(\mathrm{Ct})$ values were available for $104(64 \%)$ of unvaccinated and $36(37 \%)$ of vaccinated patients, with no significant differences between groups, table 2 .

Table 2: Characteristics of SARS-CoV-2-Positive Patients Treated with Monoclonal Antibodies by Vaccine Status Prior to Treatment 


\begin{tabular}{|c|c|c|c|}
\hline & $\begin{array}{l}\text { Non-vaccinated } \\
n(\%) \\
n=162\end{array}$ & $\begin{array}{l}\text { Vaccinated } \\
n(\%) \\
n=88\end{array}$ & P-value \\
\hline Age, median (IQR) & $39(29-47)$ & $52(41-63)$ & $<0.0001$ \\
\hline Sex & & & 0.65 \\
\hline Male & $73(45)$ & $37(42)$ & \\
\hline Female & $89(55)$ & $51(58)$ & \\
\hline Race/Ethnicity & & & 0.0002 \\
\hline Hispanic & $68(42)$ & $33(38)$ & \\
\hline Non-Hispanic Black & $69(43)$ & $20(23)$ & \\
\hline Non-Hispanic White & $11(7)$ & $20(23)$ & \\
\hline Asian & $0(0)$ & $1(1)$ & \\
\hline Other & $6(4)$ & $5(6)$ & \\
\hline Unavailable & $8(5)$ & $9(10)$ & \\
\hline BMI & $30(25-35)$ & $30(26-35)$ & 0.87 \\
\hline \multicolumn{4}{|l|}{ High-risk co-morbidities per EUA* } \\
\hline $\mathrm{BMI}>=25$ & $115(71)$ & $67(76)$ & 0.66 \\
\hline Pregnancy & $7(4)$ & $0(0)$ & 0.054 \\
\hline Chronic lung disease & $33(20)$ & $18(20)$ & 0.99 \\
\hline Chronic kidney disease & $1(1)$ & $5(6)$ & 0.0045 \\
\hline Diabetes mellitus & $22(14)$ & $25(28)$ & 0.004 \\
\hline Immunocompromised conditions** & $12(7)$ & $13(15)$ & 0.06 \\
\hline Medical-related technological dependence & $1(1)$ & $2(2)$ & 0.25 \\
\hline Neurodevelopmental disorders & $5(3)$ & $0(0)$ & 0.17 \\
\hline Cardiovascular disease or hypertension & $28(17)$ & $36(41)$ & $<0.0001$ \\
\hline Number of EUA criteria met & $1(1-2)$ & $2(1-3)$ & $<0.0001$ \\
\hline \multicolumn{4}{|l|}{ Symptom Duration Prior to Treatment } \\
\hline Days, median (IQR) & $4(3-6)$ & $3(2-5)$ & 0.02 \\
\hline Asymptomatic, n (\%) & 0 & 0 & \\
\hline
\end{tabular}


Exact days unavailable, $\mathrm{n}(\%)$

Cycle threshold (Ct) values

$<25$

Between $25-35$

$>35$

Hospitalization

All-cause 30-day admission

COVID-19-related 30-day admission

Days between vaccination and treatment, median (IQR)

Admitted (all-cause 30-day admission)

Not admitted
0

$\mathrm{n}=104$

$70(67)$

$30(29)$

4 (4)

$11(7)$

$4(5)$

0.48

7 (4)

$1(1)$
0.06

25 (69)

$6(17)$

$5(14)$

\begin{tabular}{|lcc|}
\hline Admitted (all-cause 30-day admission) & - & $154(118-166)$ \\
\hline Not admitted & - & $160(133-190)$ \\
\hline
\end{tabular}

*EUA: emergency use authorization

**Immunocompromised conditions: HIV, cirrhosis of the liver, sickle cell anemia, asplenia, malignancy, solid organ transplant, bone marrow transplant, patient received chemotherapy, chronic steroids, tacrolimus, mycophenolate, biologics, etc.

Overall, $6 \%(15 / 250)$ of patients were admitted to MMC within 30 days of mAb treatment. Eleven unvaccinated patients (7\%) were admitted (all-cause) within 30-days compared to 4 (5\%) of vaccinated patients $(p=0.48)$. One ( $1 \%)$ vaccinated patient had a COVID-19-related admission compared to $7(4 \%)$ unvaccinated patients $(p=0.27)$. When adjusting for age and number of EUA risk factors, vaccine status was still not significantly associated with either all-cause (OR:0.55, CI 0.14-1.83, $p=0.30$ ) or COVID-related 30- day admission (OR:0.19, $\mathrm{Cl} 0.01-1.71, \mathrm{p}=0.13$ ). Median duration between vaccination and $\mathrm{mAb}$ treatment was 160.5 days; 153.5 days for admitted and 160.5 days for non-admitted patients.

\section{Discussion}

Although low absolute admission numbers, mAb recipients had a similar all-cause 30 -day admission rate regardless of vaccination status supporting FDA in-vitro data indicating retained activity of casirivimab/imdevimab against B.1.617.2. ${ }^{14}$ Among the 250 treated patients, the majority were unvaccinated, which reflects local statistics in the Bronx during this timeframe. ${ }^{13}$ One vaccinated and 7 unvaccinated patients were admitted for worsening COVID-19.

Overall admissions in this cohort were lower and median age was younger compared to patients treated at our medical center in early $2021^{8}$, likely due to expanded EUA criteria allowing treatment of younger patients with fewer comorbidities. A low rate of admissions among vaccinated individuals suggests that 
prioritization of unvaccinated or higher risk vaccinated individuals (e.g., age $>65$ with more comorbidities, potentially poor vaccine response, or extended interval since last vaccination) is supported, given recent modifications to the federal mAb allocation process. ${ }^{16}$

Our findings indicate that vaccinated, treated patients were more likely to be older and have more underlying risk factors for development of severe COVID-19. Despite this, vaccinated patients had a low rate of both all-cause (5\%) and COVID-related (1\%) admission within 30-days, which is likely due to the well documented effectiveness of vaccines in protecting against severe disease, hospitalization, and death. ${ }^{17-22}$ Data from hospitals across the country indicate that most patients admitted with COVID-19 were unvaccinated during the study timeframe. ${ }^{23-25} \mathrm{~A}$ study of fully vaccinated mAb-treated patients reported that the number needed to treat (NNT) to prevent one hospitalization was 225 among the lowestrisk patients compared to a NNT of 4 among the highest risk patients. ${ }^{26}$ Our results also suggest that a "watch and wait" approach is appropriate for fully vaccinated patients with fewer comorbidities versus an immediate test and treat approach for the highest risk patients.

Median duration between vaccination and mAb treatment was high in both admitted and non-admitted patients at 154 and 161 days, respectively, suggesting that waning vaccination-induced antibody levels may have contributed to "breakthrough" infections in our cohort.

Overall, 30-day admission for mAb untreated, SARS-CoV-2 positive patients at MMC (regardless of vaccine status) was low at $4 \%(22 / 538)$ between July-August 2021, perhaps due to a high background rate of prior infection (seroprevalence of $24.3 \%$ ) along with steadily increasing uptake of vaccination. ${ }^{27}$ The overall admission rate of mAb-treated patients was similarly low at $6 \%$. Lieberman-Cribbin and colleagues reported that the Bronx residents are more likely to have increased SARS-CoV-2 seropositivity suggestive of past infection. ${ }^{27}$

Acharya and colleagues demonstrated that there is no significant difference in viral load between vaccinated and unvaccinated patients infected with the SARS-CoV-2 Delta variant. ${ }^{28}$ We also did not observe any significant difference in available $\mathrm{Ct}$ values between vaccinated and unvaccinated patients, although few vaccinated patients had documented $\mathrm{Ct}$ values since most were tested outside of our system. A limitation of our study is that the sample size was only 250 patients with full 30-day follow up, and other mAb-treatment outcomes such as reduction in symptom severity or duration, or spread to secondary contacts, were not evaluated. Additionally, the observational, non-randomized, design limited the ability to draw further conclusions about vaccinated vs. unvaccinated mAb-treatment outcomes. The current unprecedented Omicron surge has intensified the resource scarcity of mAbs and the need to prioritize patients at highest risk of hospitalization and death. Institutional prioritization criteria utilized during the Delta wave (table 1) were tightened significantly December 2021 onward.

\section{Conclusion}


Since initial authorization of SARS-CoV-2 mAb therapies, multiple factors have impacted the effectiveness of mAbs, including local access to care and SARS-CoV-2 variants. Limited federal allocation of mAbs compels infusion programs to prioritize patients at highest risk of death from COVID19 , particularly during the current Omicron surge. We suspect that the role of mAb therapies in COVID-19 treatment and prevention armamentarium will continue to evolve as other outpatient therapies become available.

\section{Declarations}

\section{Ethics approval and consent to participate}

The study was approved by Albert Einstein College of Medicine institutional review board approved with waiver of informed consent since analyses were based on retrospective data collection via electronic health record (IRB\# 2020-12525). All methods were performed in accordance with the relevant guidelines and regulations as stated in the Declarations of Helsinki.

\section{Consent for publication}

Not applicable

\section{Availability of data and materials}

The datasets generated and/or analyzed during the current study are not publicly available due to patient privacy and confidentiality but are available from the corresponding author on reasonable request.

\section{Competing interests}

PN receives speaker fees from Regeneron and Medscape. All other authors declare that they have no competing interests.

\section{Funding}

This research did not receive any specific grant from funding agencies in the public, commercial, or notfor-profit sectors.

\section{Authors' contributions}

YG, KC, MC, HB, AG, TM, LB, RS, LP, PN contributed to study design, data review, and manuscript preparations. $Y G, M C, H B, A G, T M$ also completed data collection. $K C$ and EA performed software data acquisition and interpretation of data. KC completed the formal statistical analysis. YG, KC, PN drafted manuscript. All authors reviewed and edited manuscript. All authors have read and approved the final manuscript.

\section{Acknowledgements}




\section{References}

1. Gottlieb RL, Nirula A, Chen P, et al. Effect of Bamlanivimab as Monotherapy or in Combination with Etesevimab on Viral Load in Patients with Mild to Moderate CVID-19: a Randomized Clinical Trial. JAMA 2021;325(7):632-44

2. Weinreich DM, Sivapalasingam s, Norton T, et al. REGN-COV2, a Neutralizing Antibody Cocktail, in Outpatients with COVID-19. N Engl J Med 2021;384(3):238-51

3. Jenks JD, Aslam S, Horton LE, et al. Early Monoclonal Antibody Administration can Reduce Both Hospitalization and Mortality in High-risk Outpatients with COVID-19. Clin Infect Dis. 2021 Jun 6:ciab522. doi: 10.1093/cid/ciab522.

4. Kumar RN, Wu EL, Stosor V, et al. Real-world Experience of Bamlanivimab for COVID-19: a Casecontrol Study. Clin Infect Dis. 2022 Jan 7;74(1):24-31.

5. Ganesh R, Pawlowski C, O'Horo JC, et al. Association of Intravenous Bamlanivimab Use with Reduced Hospitalization, Intensive Care Unit Admission, and Morality in Patients with Mild to Moderate COVID-19. MedRxiv 2021 May 25:2021.05.23.21257670. doi:

10.1101/2021.05.23.21257670.

6. Bariola JR, McCreary E, Wadas RJ, et al. Impact of Bamlanivimab Monoclonal Antibody Treatment on Hospitalized and Mortality among Nonhospitalized Adults with Severe Acute Respiratory Syndrome Coronavirus 2 Infection. Open Forum Infect Dis. 2021 May 17;8(7):ofab254. doi: 10.1093/ofid/ofab254.

7. Cooper MH, Christensen PA, Salazar E, et al. Real-world Assessment of 2879 COVID-19 Patients Treated with Monoclonal Antibody Therapy: a Propensity Score-matched Cohort Study. Open Forum Infect Dis. 2021;8:ofab512, https://doi.org/10.1093/ofid/ofab512

8. Cowman K, Guo Y, Pirofski L, et al. Post-severe Acute Respiratory Syndrome Coronavirus 2 Monoclonal Antibody Treatment Hospitalizations as a Sentinel for Emergence of Viral Variants in New York City. Open Forum Infect Dis. 2021 Jun 12;8(8):ofab313. doi: 10.1093/ofid/ofab313.

9. New York City Department of Health COVID-19: Data https://www1.nyc.gov/site/doh/covid/covid-19data-variants.page Assessed 30 December 2021

10. Bergwerk M, Gonen T, Lustig Y, et al. Covid-19 Breakthrough Infections in Vaccinated Health Care Workers. N Engl J Med. 2021 Oct 14;385(16):1474-1484.

11. New York State Department of Health COVID-19 Breakthrough Data https://coronavirus.health.ny.gov/covid-19-breakthrough-data Assessed 14 December 2021

12. Levin EG, Lustig Y, Cohen C, et al. Waning Immune Humoral Response to BNT162b2 Covid-19 Vaccine over 6 Months. N Engl J Med. 2021 Oct 6:NEJMoa2114583. doi: 10.1056/NEJMoa2114583.

13. New York City Department of Health COVID-19: Data https://www1.nyc.gov/site/doh/covid/covid-19data-vaccines.page\#people Assessed 14 December 2021 
14. Fact sheet for health care providers emergency use authorization (EUA) of Regen-COV (casirivimab and imdevimab). https://www.fda.gov/media/145611/download Assessed 14 December 2021

15. Fact sheet for health care providers emergency use authorization ((EUA) of bamlanivimab and etesevimab. https://www.fda.gov/media/145802/download Assessed 14 December 2021

16. HHS announces state/territory-coordinated distribution system for monoclonal antibody therapeutics. https://www.phe.gov/emergency/events/COVID19/investigation-MCM/Bamlanivimabetesevimab/Pages/Update-13Sept21.aspx Assessed 14 December 2021

17. Polack FP, Thomas SJ, Kitchin N, et al. C4591001 Clinical Trial Group. Safety and Efficacy of the BNT162b2 mRNA Covid-19 Vaccine. N Engl J Med. 2020 Dec 31;383(27):2603-2615.

18. Thompson MG, Stenehjem E, Grannis S,et al. Effectiveness of Covid-19 Vaccines in Ambulatory and Inpatient Care Settings. N Engl J Med. 2021 Oct 7;385(15):1355-1371.

19. Pilishvili T, Gierke R, Fleming-Dutra KE, et al. Vaccine Effectiveness among Healthcare Personnel Study Team. Effectiveness of mRNA Covid-19 Vaccine among U.S. Health Care Personnel. N Engl J Med. 2021 Dec 16;385(25):e90.doi: 10.1056/NEJMoa2106599.

20. Pilishvili T, Fleming-Dutra KE, Farrar JL, et al. Interim Estimates of Vaccine Effectiveness of PfizerBioNTech and Moderna COVID-19 Vaccines Among Health Care Personnel - 33 U.S. Sites, JanuaryMarch 2021. MMWR Morb Mortal Wkly Rep May 21, 2021/70(20);753-758

21. Self WH, Tenforde MW, Rhoads JP, et al. Effectiveness of Pfizer-BioNTech and Moderna Vaccines Against COVID-19 Among Hospitalized Adults Aged $\geq 65$ Years - United States, January-March 2021. MMWR Morb Mortal Wkly Rep May 7, 2021/70(18);674-679

22. Thompson MG, Bergess JL, Naleway AL, et al. Interim Estimates of Vaccine Effectiveness of BNT162b2 and mRNA-1273 COVID-19 Vaccines in Preventing SARS-CoV-2 Infection Among Health Care Personnel, First Responders, and Other Essential and Frontline Workers - Eight U.S. Locations, December 2020-March 2021. MMWR Morb Mortal Wkly Rep April 2, 2021/70(13);495-500

23. Rosenberg ES, Holtgrave DR, Dorabawila V, et al. New COVID-19 Cases and Hospitalizations Among Adults, by Vaccination Status - New York, May 3-July 25, 2021. MMWR Morb Mortal Wkly Rep September 17, 2021/70(37);1306-1311

24. Lacobucci G. Covid-19: how is vaccination affecting hospital admissions and deaths? BMJ. 2021 Sep 20;374:n2306. doi: 10.1136/bmj.n2306.

25. Havers FP, Pham H, Taylor CA, et al. COVID-19 associated hospitalizations among vaccinated and unvaccinated adults $>18$ years-COVID-NET, 13 states, January 1-July 24,2021 . medRxiv preprint doi: https://doi.org/10.1101/2021.08.27.21262356

26. Bierle DM, Ganesh R, Tulledge-Scheitel S, et al. Monoclonal Antibody Treatment of Breakthrough COVID-19 in Fully Vaccinated Individuals with High-Risk Comorbidities, J Infect Dis. 2021 Nov 16;jiab570. doi: 10.1093/infdis/jiab570.

27. Lieberman-Cribbin W, Galanti M, Shaman J. Socioeconomic Disparities in SARS-CoV-2 Serological Testing and Positivity in New York City, Open Forum Infect Dis. 2021 Oct 17;8(12):ofab534. doi: 10.1093/ofid/ofab534. 
28. Acharya CB, Schrom J, Mitchell AM, et al. No Significant Difference in Viral Load between Vaccinated and Unvaccinated, Asymptomatic and Symptomatic Groups when Infected with SARS-CoV-2 Delta Variant. medRxiv preprint doi: https://doi.org/10.1101/2021.09.28.21264262 